\title{
Parent-Implemented Behavior Interventions via Telehealth for Older Children and Adolescents
}

\author{
Christine M. Drew ${ }^{1}$ D $\cdot$ Wendy Machalicek ${ }^{2} \cdot{\text { Becky } \text { Crowe }^{2} \text { (D) }}^{\text {. }}$ \\ Lindsay Glugatch ${ }^{2} \cdot$ Qi Wei $^{2} \cdot$ Buket Erturk $^{2}$
}

\section{Accepted: 4 November 2021}

(C) The Author(s), under exclusive licence to Springer Science+Business Media, LLC, part of Springer Nature 2021

\begin{abstract}
Children and adolescents with intellectual and developmental disabilities (IDD) are more likely to engage in challenging and interfering behavior than their typically developing peers, which has been linked to many negative outcomes. The most effective interventions to address challenging and interfering behavior incorporate function-based assessments, which are used to develop individualized behavior interventions. Functional communication training (FCT) is an evidence-based practice to decrease challenging and interfering behavior that can be taught to parents using behavioral parent training (BPT); however, there are limited skilled professionals who can develop interventions and train parents. Telehealth can enable greater access to these professionals. This study used withdrawal designs to determine whether high parent treatment fidelity resulted in decreased challenging and interfering behavior and increased appropriate replacement behavior. Three participants (8-17 years) were included in the study, and their parents served as interventionists during mealtime, toothbrushing, and room cleaning. Data were analyzed using visual analysis. Each parent achieved high treatment fidelity with one session of BPT and bug-in-ear coaching. All participants had a decrease in challenging and interfering behavior and an increase in functional communication responses (FCRs) upon the introduction of the intervention with reliable reversals. All parents reported high social validity. Results and implications for practice and future research are discussed.
\end{abstract}

Keywords Challenging behavior · Parent-implemented · Telehealth · Adolescents · Developmental disability

Christine M. Drew

cmd0109@auburn.edu

1 Department of Special Education, Rehabilitation, and Counseling, Auburn University, Haley Center 2084, Auburn, AL 36849, USA

2 Department of Special Education and Clinical Sciences, University of Oregon, Eugene, OR, USA 


\section{Introduction}

Individuals with intellectual disability (ID) are at risk for developing challenging and interfering behavior (Simó-Pinatella et al., 2019). Challenging behavior is any behavior that impacts a person's ability to learn social or academic skills or engage with peers or others (Emerson, 2001). Broadly, this can also include interfering behavior such as stereotypy and noncompliance (Gentry \& Luiselli, 2008). Challenging and interfering behavior puts them at risk for less participation in religious services, school, and organized activities (Lee et al., 2008). Challenging behavior can also increase the use of medications like antipsychotics (Matson \& Neal, 2009). Challenging behavior can persist across the lifespan into adulthood (O'Dwyer et al., 2018). The estimated prevalence of aggression in the intellectual disability population varies widely from $10 \%$ to $71.5 \%$ (Simó-Pinatella et al., 2019). A population survey across the lifespan found that $10 \%$ of individuals with intellectual disability engaged in uncontrolled challenging behavior that caused harm to self or others (Lowe et al., 2007).

The most effective way to address challenging and interfering behavior is functional assessment and behavioral interventions (Wong et al., 2015). Functional assessments provide the best hypothesis for the operant function of an individual's challenging and interfering behavior and function-based behavioral interventions are more effective than non-function-based interventions (Machalicek et al., 2016; Matson, 2012). One of the most effective function-based intervention to address challenging and interfering behavior is functional communication training (FCT). FCT consists of providing reinforcement for a functional communication response (FCR) to obtain the same consequence as challenging and interfering behavior. FCT is an effective intervention to reduce challenging and interfering behavior and teach and reinforce communication skills. Natural change agents, including parents, can serve as effective interventionists for teaching and reinforcing communication responses (FCRs) and decreasing challenging and interfering behavior (Hanley et al., 2014; Wong et al., 2015).

In spite of the evidence to support the use of function-based assessments and specifically FCT, there are substantial barriers to obtaining services. One barrier is the limited number of professionals who are trained in behavioral interventions and have the skills to carry out parent training. The majority of these professionals are also concentrated in urban areas, which may place an additional burden on families who live in rural areas (Kogan et al., 2008). One survey found that rural residents reported driving longer distances and experiencing longer wait times to access services than their non-rural peers. Rural residents also reported lower utilization of behavior supports in home and school as well as parent supports like workshops, support groups, or speaking with other parents (Mello et al., 2016).

One strategy for increasing access to evidence-based practices for challenging and interfering behavior is the use of telehealth. Previous research has shown that telehealth for young children with autism spectrum disorder (ASD) is more cost-effective than in-home applied behavior analysis (ABA) services and is just as successful at decreasing challenging behavior and maintaining 
high parent social validity ratings (Lindgren et al., 2016).Telehealth has been used in many fields to address shortages of qualified personnel, provide supervision to medical, mental health, and educational professionals, and assist individuals needing support with complex procedures (Hilty et al., 2013). There is a substantial amount of research on the use of telehealth to address challenging behavior for individuals with ASD and IDD, but it has also primarily focused on young children (Unholz-Bowden et al., 2020; Wacker et al., 2013). A recent systematic review found 28 studies involving caregiver training via telehealth to address challenging behavior. Of the 157 participants, only two were adolescents (Unholz-Bowden et al., 2020). One study used BST via telehealth to train special educators in functional analysis methodology. The 12-year-old participant was only included in the generalization phase of the study (Alnemary et al., 2015). The other study included two children and one adolescent with ASD. The adolescent participant, Emma, engaged in self-injurious behavior and inappropriate non-word vocalizations (Machalicek et al., 2016). The authors noted that Emma's challenging behavior did not result in injury to her or her parent throughout the study. Parents were coached via telehealth to complete functional analysis and treatment comparison sessions. Emma's challenging behavior decreased to zero levels for both interventions.

One instructional method that can be used to ensure that natural change agents like parents achieve high treatment fidelity while implementing these interventions is behavioral parent training (BPT). BPT is behavior skills training used with parents and includes four components: didactic instruction, modeling of targeted skills, rehearsal of targeted skills using role play and with child, and performance feedback (Dib \& Sturmey, 2012). Research into the use of BPT has shown that parents are able to attain high levels of treatment fidelity for many interventions and clinically significant decreases in child challenging behavior. Many studies have assessed the use of BPT for young children with ASD/ IDD both in vivo and via telehealth (e.g., Machalicek et al., 2015, 2016). There is limited research addressing the use of BPT with the parents of older children with IDD via telehealth. While parent-implemented FCT has been shown to be an evidence-based practice for young children and has been delivered via telehealth, older children and adolescents are largely absent from this research (Suess et al., 2014). This research expanded the literature by including older children and adolescents as participants and conducting the intervention via telehealth. We aim to answer the following research questions:

1. What is the effect of increased parent treatment fidelity of function-based interventions (adherence to task analysis of intervention steps) on target challenging and interfering behavior displayed by a child with IDD during naturally occurring routines?

2. What is the effect of increased parent treatment fidelity of a function-based intervention (adherence to task analysis of intervention steps) on the level of adaptive/ replacement behavior displayed by a child with IDD during naturally occurring routines? 


\section{Method}

\section{Participants and Settings}

All participants were recruited from the office of a developmental behavioral pediatrician that served children and adolescents with disabilities in the region. Joe was a 15-year-old white male with ASD and ID. He communicated using idiosyncratic signs, one-word verbal utterances, and gestures. Gina, his mother was a White female who worked part time as a registered nurse. Lucas was a 17-year-old multiracial (Black and White) male with ASD, ID, and fetal alcohol syndrome. He communicated using vocal verbal speech including full sentences to meet his daily wants and needs. Catie, Lucas mother, was a White female who was unemployed during the study. She had finished high school. Henry was an 8-year-old white male with ASD, ID, hearing loss, and a speech disorder. He communicated with single manual signs and relied primarily on gestures and non-word utterances. Henry also engaged in aggression and self-injury to communicate his wants and needs. Daniel, Henry's mother's long-term partner, was a White male and his education consisted of vocational training. Daniel typically attempted the toothbrushing routine with Henry, so Daniel took on the role of interventionist. None of the parent interventionists reported previous training on challenging or interfering behavior or the use of behavioral strategies. All sessions took place in family home settings (kitchen, bedroom, and bathroom). None of the families were receiving behavioral services through school or an outside provider at the time of the study. Both Joe and Lucas had received services in the past for challenging behavior, but parents reported that the services did not result in behavior change. Henry's mother had received some consultation in the past that did not specifically address challenging behavior. ABA services are not widely available in the geographic region included in the study. One family lived in a rural area and two others lived in a town of less than 65,000 people and none of the families had access to ABA services. The researcher conducted all sessions on a university campus in a clinic office. The participants lived an average of 30 miles (range $=11-68$ miles) from the university (Table 1 ).

\section{Researcher}

The researcher was a White female Board Certified Behavior Analyst (BCBA) with 5 years' experience teaching children/adolescents with IDD in public school and experience serving as primary interventionist in previous studies addressing challenging behavior.

\section{Dependent Variables and Response Measurement}

Dependent variables were (a) child challenging and interfering behavior (10-s partial interval); (b) prompted and independent functional communication responses (frequency); (c) appropriate behavior (Lucas [percentage of task 
Table 1 Parent Characteristics

\begin{tabular}{lllll}
\hline Name & Race & Gender & Occupation & Relationship to Participant \\
\hline Gina & White & F & Surgical nurse & Mother \\
Catie & White & F & Unemployed & Mother \\
Daniel & White & M & Woodworker & Father \\
Kelley & White & F & Housekeeping & Mother \\
& & & supervisor & \\
\hline
\end{tabular}

analysis completed] and Henry [duration of toothbrush placed into mouth]), and (d) parent treatment fidelity (percentage of correct responding). For complete descriptions of child dependent variables, refer to Table 2. Parent treatment fidelity consisted of defined steps for each parent from their child's behavior support plan (BSP). For each session, parent completion of steps of the BSP were scored on a scale of $0-3$ or not applicable. The scores of $0-3$ correspond to percent of parent correct opportunities to respond. The scores of the number of total steps were divided by the number of possible points for items completed and multiplied by 100 to obtain a percentage ranging from 0 to $100 \%$ of steps completed correctly. Scoring was based on specific parent behavior (ex: $0=$ parent completed tasks for $0-25 \%$ of opportunities, $1=$ parent completed tasks for $26-50 \%$ of opportunities, $2=$ parent completed tasks for $51-75 \%$ of opportunities, $3=$ parent completed task for $76-100 \%$ of opportunities). Only independent parent responding was scored.

\section{Target Routines and Child Behavior}

Gina (Joe's mother) selected the family dinner as the routine which consisted of a prayer, serving food, family conversation, eating, and then asking to leave the table. The interfering behavior targeted were: eating with hands, physical contact with his mother and furniture, touching others' plates and food items, and turning away from the table. Catie (Lucas mother) selected the room cleaning routine that occurred after school each day during which Lucas was asked to make his bed, clean up plates and food wrappers, pick up dirty laundry, put away clean laundry, and sweep the floor. The behavior targeted was negative vocalizations which included saying no, screaming, yelling, making threats, growling, screeching, and telling his mother what to do. Kelley and Daniel (Henry's mother and father) decided to address the toothbrushing routine which took place at least once a day before bedtime and included walking to the bathroom and allowing his father to brush his teeth. Henry's challenging behavior included noncompliance, self-injury, and aggression. Operational definitions of challenging and behavior included in Table 2. 


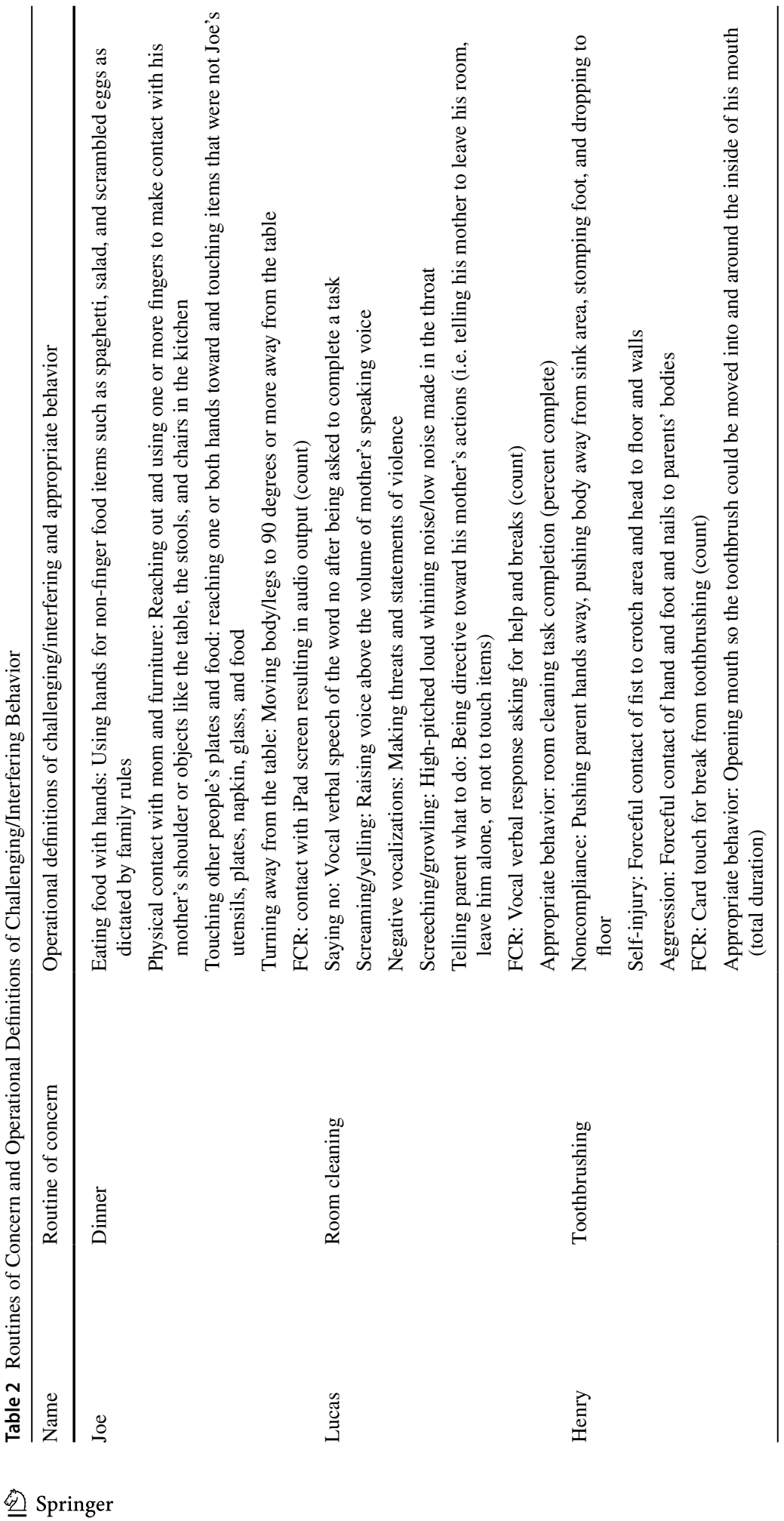




\section{Hardware and Software}

The researcher used a 13-inch 2017 MacBook Pro ${ }^{\mathrm{TM}}$ laptop with $3.1 \mathrm{GHz}$ Intel Core i5 and 8 GB of DDR3 memory, and all parents used their personal Apple ${ }^{\mathrm{TM}}$ smart phones. The researcher provided an AMINYTM UFO Bluetooth ${ }^{\mathrm{TM}}$ headset to each parent. No additional hardware was required. Videoconferencing calls were conducted using the free version of VSee software (http://vsee.com). This software is federal Health Insurance Portability and Accountability Act of 1996 (HIPAA), Pub. L. 104-191, 42 U.S.C. §§ 1320d et seq. compliant.

\section{Study Design}

This study consisted of (a) functional behavior assessment (FBA) and intervention creation, (b) experimental evaluation of the intervention using an A-B-A-B withdrawal design, and (c) pre- and post-intervention social validity assessment. Data were analyzed using visual analysis following single case visual analysis guidelines (Ledford \& Gast, 2018).

\section{Procedures}

\section{Behavioral Parent Training (BPT)}

Each parent was trained using BPT: didactic instruction, modeling, rehearsal, and feedback (Dib \& Sturmey, 2012). The didactic instruction included a PowerPoint ${ }^{\mathrm{TM}}$ presentation via screenshare, written intervention steps, and rationale for the intervention. The researcher modeled the intervention by demonstrating all of the parents' steps of the BSP with the parent playing the part of the child (e.g., collecting task analysis and providing praise for appropriate behavior for Lucas), and the researcher provided narration of actions and referred the parent treatment fidelity checklist. The researcher then role played with the parent engaging in the tasks on the treatment fidelity checklist using all materials and the researcher playing the part of the child. If the parent engaged in an incorrect response, the researcher provided immediate verbal prompts for the appropriate response and referred the parent back to the treatment fidelity checklist. Once the parent performed the BSP steps independently with $100 \%$ treatment fidelity, they completed a modified version of the Treatment Acceptability Rating Form-Revised (TARFR; Reimers \& Wacker, 1988), which asked parents to rate the acceptability of the procedures of the BSP. The training took on average $56 \mathrm{~m} 11 \mathrm{~s}($ range $=49 \mathrm{~m} 36 \mathrm{~s}$ $-59 \mathrm{~m} 56 \mathrm{~s}$ ) and each parent reached mastery in 1 session. 


\section{Parent-implemented Intervention with Coaching Support}

Prior to the start of each routine, the researcher reminded the parent to gather all materials needed for the BSP. During sessions, the researcher provided verbal prompts on a 0-s time delay via the headset worn by the parent if the parent incorrectly implemented an intervention strategy. The verbal prompts consisted of positively-stated steps of the intervention (e.g., "Remember to ask if he wants a break or help if he yells.") The researcher provided behavior-specific praise when a parent engaged in a behavior that was part of the BSP. If the parent waited longer than $2 \mathrm{~s}$ after a response was appropriate, the researcher used a verbal prompt as described above.

\section{Preassessment}

The Functional Assessment Interview - Adolescent (FAI) and a 25-item Questions About Behavioral Function (QABF) were completed with the participating parent via telehealth (Matson \& Vollmer, 1995; O'Neil et al., 1997). The QABF has the strongest psychometric properties of assessments available and the implementation of function-based interventions derived from the results of the QABF have shown statistically significant improvements compared to a standardized control condition (Matson et al., 2012; 1999). Results of the QABF correspond with the results of experimental functional analyses across participant diagnoses, topographies of challenging behavior, and function (Healy et al., 2013). This study was conducted remotely via telehealth and there are ethical and safety considerations for having parents implement functional analysis procedures with older children and adolescents which result in temporary increases in challenging behavior. Based on parent report of occasional engagement in self-injury and aggression during the FAI, a session termination criterion was created for Henry prior to the first baseline session.

The results of the interviews indicated the potential for challenging behavior maintained by (a) positive reinforcement in the form of access to adult attention or tangibles for Joe and (b) negative reinforcement in the form of avoidance or escape from demands for Lucas and Henry. The hypothesized functions from the FAI were corroborated by the QABF scores. For participant functional assessment results, age, race, and gender (Table 3).

Table 3 Child Characteristics

\begin{tabular}{lllll}
\hline Name & Race & Gender & Age & $\begin{array}{l}\text { Function } \\
\text { of Chal- } \\
\text { lenging } \\
\text { Behavior }\end{array}$ \\
\hline Joe & White & $\mathrm{M}$ & 15 & $\begin{array}{l}\text { Attention } \\
\text { and Tan- } \\
\text { gible }\end{array}$ \\
Lucas & White and Black & $\mathrm{M}$ & 17 & $\begin{array}{l}\text { Escape } \\
\text { Escape }\end{array}$ \\
\hline
\end{tabular}




\section{Baseline}

At the start of each baseline session, the researcher provided a verbal overview of the baseline procedures. Baseline phase was structured for all participants. During this phase, no stimuli associated with the behavior intervention plan (e.g., task analysis, visuals) were present. The parent began the routine of concern with a specific antecedent (e.g., "Time to clean your room." or "Time to brush your teeth."). The parent completed the entire routine (Joe) or re-presented the antecedent multiple times (Lucas and Henry). Joe's routine was family dinner, so the entire routine was usually completed with family members ignoring Joe's interfering behavior. For Lucas and Henry, prior to the researcher's observation, the routines were not being completed and the parents reported that they would present the antecedent multiple times, without success. The researcher provided prompts as needed via the headset (e.g., "Say "Time to brush teeth"). With both Henry and Lucas, any instance of challenging or interfering behavior resulted in the temporary removal of the demand (30 s). With Joe, interfering behavior resulted in disapproving verbal behavior from Gina (e.g., "I don't like when you eat with your hands! That's not how we eat!") as that was her typical response. During withdrawal sessions, each parent was instructed to return to the same behavior as baseline. No stimuli associated with the BSP were present including materials needed for FCR (Henry and Joe), the parents did not use any antecedent, teaching, or reinforcement procedures. There was no training provided to parents for baseline and withdrawal phases and all prompts were provided via bug-in-ear coaching from the researcher in real time.

\section{Individualized BSP}

The researcher worked collaboratively with each parent to develop a multicomponent behavior support plan (BSP) based on the results of the QABF and contextual variables gathered during the FAI-Adolescent. Each plan included antecedent, teaching, and consequence interventions and a core component of FCT. FCT has the strongest research base as an intervention for challenging behavior and has been used effectively by parents in home settings (Gerow et al., 2018). FCRs were selected based on the communication responses parents reported during the FAI. For example, Lucas spoke in complete sentences, so simple verbal FCRs of "help" and "break" were selected. Reponses were selected to require less effort than the current challenging behavior.

Joe. The BSP for Joe included a review of expectations for family dinner and role play of the use of an $\mathrm{iPad}^{\mathrm{TM}}$ with the communication application GoTalk NOW Lite iPad® (Attainment Company). Before dinner, Gina would follow a script with Joe. She would then ask him to show her how to use each of the buttons on his device. She also reviewed expectations for dinner (i.e., "First we pray, then we eat."). Gina provided reinforcement for FCRs ("help", "more", and "I have something to say.") by providing assistance with using a fork for "help", more of food or drink items for "more", and turning to Joe and saying "I hear you! What do you want to say?" for 
"I have something to say." Gina also prompted Joe to engage in FCRs if interfering behavior occurred. Joe was taught to emit FCRs using an $\mathrm{iPad}^{\mathrm{TM}}$ with the communication application GoTalk NOW Lite iPad® (Attainment Company) over 4 teaching sessions during snack time prior to the first intervention session using most-toleast prompting. Guided access was turned on to prevent Joe from using other iPad® functions. Joe's BSP had 8 steps, 4 of which took place before dinner began (collecting all needed items, modeling FCRs, testing FCRs, and review of rules), 3 of which took place during dinner (parent talking to other family members, reinforcement of FCRs on an FR1 schedule, and prompts to engage in FCRs if interfering behavior occurred [extinction]), and 1 for after dinner (positive verbal interactions about dinner). Data were collected for the first $5 \mathrm{~min}$ of the dinner routine.

Lucas. The BSP for Lucas included environmental arrangement (having the broom and other cleaning supplies upstairs near Lucas room), the use of a task analysis checklist filled out by Catie, verbal praise for each task completed, the opportunity to take up to three breaks and request help with up to three tasks (FCRs), reminders about FCRs if interfering behavior occurred ("Would you like to take a break?" or "You can ask me for help if you're having trouble.") and tangible reinforcement for completing the routine (access to video games and cell phone). Lucas was taught the steps for completing room cleaning using the task analysis in 2 sessions prior to the first intervention session. Lucas behavior support plan had 12 steps, 4 of which occurred before room cleaning began (gathering all items needed for the routine, beginning the routine for after Lucas took his afternoon medications, modeling and role play of using FCRs, and reminders of reinforcement contingencies), 6 that occurred during the routine (checking of items on the task analysis for room cleaning, providing brief verbal praise for each item completed, reinforcement of FCRs on an FR1 schedule for up to 3 FCRs, reminders of FCRs if interfering behavior occurred [extinction], redirection to task analysis if all FCRs had been used, and reminders of the availability of games or cell phone once tasks were complete), and 2 for after the room cleaning was complete (providing him with his phone or games, and leaving the room). Data were collected for the first $10 \mathrm{~min}$ of the room cleaning routine.

Henry. The BSP for Henry included reminders of contingencies, prompting to use the break card if he engaged in challenging behavior (FCR), and a token economy with tangible reinforcement (access to vacuum). The behavior intervention plan for Henry included choices between toothbrushes of different colors and designs, reminders of contingencies such as "If you want to play with the vacuum, we need to brush teeth" and "If you want a break touch the break card." He was physically prompted to use the break card to mand for a break from toothbrushing if he engaged in challenging behavior, and Daniel implemented token economy for Henry to earn preferred activities. One token was earned for every $5 \mathrm{~s}$ the toothbrush remained in Henry's mouth. After 5 tokens, Henry gained access to the vacuum. FCRs resulted in $30 \mathrm{~s}$ break from the toothbrushing routine. Henry remained in the bathroom and was allowed to sit on the floor or move away from the sink area. Before the first intervention session, Henry was taught to use the token economy and token board to earn preferred items and to use a break card FCR to mand for ceasing the routine for $15 \mathrm{~s}$ in the context of an unpreferred task (wearing hearing aids). Henry met 
mastery criterion in 7 sessions. Henry was the only participant that engaged in challenging behavior that posed a potential harm to himself or others. To address this, Daniel was instructed to block attempts of aggression or self-injury. Daniel and the researcher also established a termination criterion, which was 3 instances of aggression or self-injury in a session. No sessions met termination criteria. Henry's BSP had 12 steps, 4 of which took place before the routine began: gathering materials, showing Henry the break card and tokens, verbal reminder of contingencies, and prompts (least-to-most) to go to the bathroom, 7 during the routine (letting Henry choose a toothbrush, placing the toothbrush in his mouth, immediate verbal praise for opening mouth, counting down from 5 out loud, placing a token on the board after $5 \mathrm{~s}$, providing breaks for FCRs, and prompting the use of FCRs if challenging behavior occurred [extinction]), and 1 after the routine (provide access to the vacuum cleaner). Data were collected for the first $5 \mathrm{~min}$ of the toothbrushing routine.

\section{Interobserver Agreement}

IOA data were collected for $38.88 \%$ of baseline/withdrawal sessions (range $=29$ $-40 \%$ ) and $36 \%$ of intervention sessions (range $=33-40 \%$ ) across dyads. Secondary independent data collectors collected reliability data by watching recorded sessions. IOA was calculated point-by-point, meaning that items with disagreement were subtracted from items with agreement, divided by all items and multiplied by 100 (Ledford \& Gast, 2018). IOA for challenging and interfering behavior was: $92 \%$ (range $=88-94 \%$ ) for Joe, 93\% (range $=82-98 \%$ ) for Lucas, and 95\% (92-98\%) for Daniel. IOA was $100 \%$ for FCRs for all participants. IOA for parent treatment fidelity was 90\% (range $=82-95 \%$ ) for Gina, 94\% (range $=90$ - 100\%) for Catie, and 88\% (range $=85-96 \%$ ) for Daniel. For Lucas, IOA for task analysis completion was $99 \%$ (range $=97-100 \%$ ), and for Daniel, IOA for time spent with toothbrush in mouth was $91 \%$ (range $=85-96 \%$ ).

\section{Researcher Procedural Fidelity}

Researcher procedural fidelity was assessed for each phase of the research asynchronously via video recording: interviews/preassessments (QABF/FAI), behavioral parent training (BPT), and coaching by trained data collectors (special education doctoral students) using fidelity checklists. For the interview/preassessments, the researcher had $96 \%$ procedural fidelity (range $=85-100 \%$ ). IOA on procedural fidelity was assessed for $38 \%$ of sessions (range $=36-40 \%$ ) and was $95 \%$ (range $=88-100 \%$ ). For the BPT sessions, procedural fidelity was assessed for $100 \%$ of sessions and was $93 \%$ (range $=85-100 \%$ ). IOA on procedural fidelity was assessed for $33 \%$ of sessions and was $96 \%$. Procedural fidelity for coaching was $96 \%$ (range $=80-100 \%$ ) and assessed for $36 \%$ of sessions. IOA on coaching was $90 \%$ (range $=80-99 \%$ ) and conducted on $46 \%$ of sessions. 


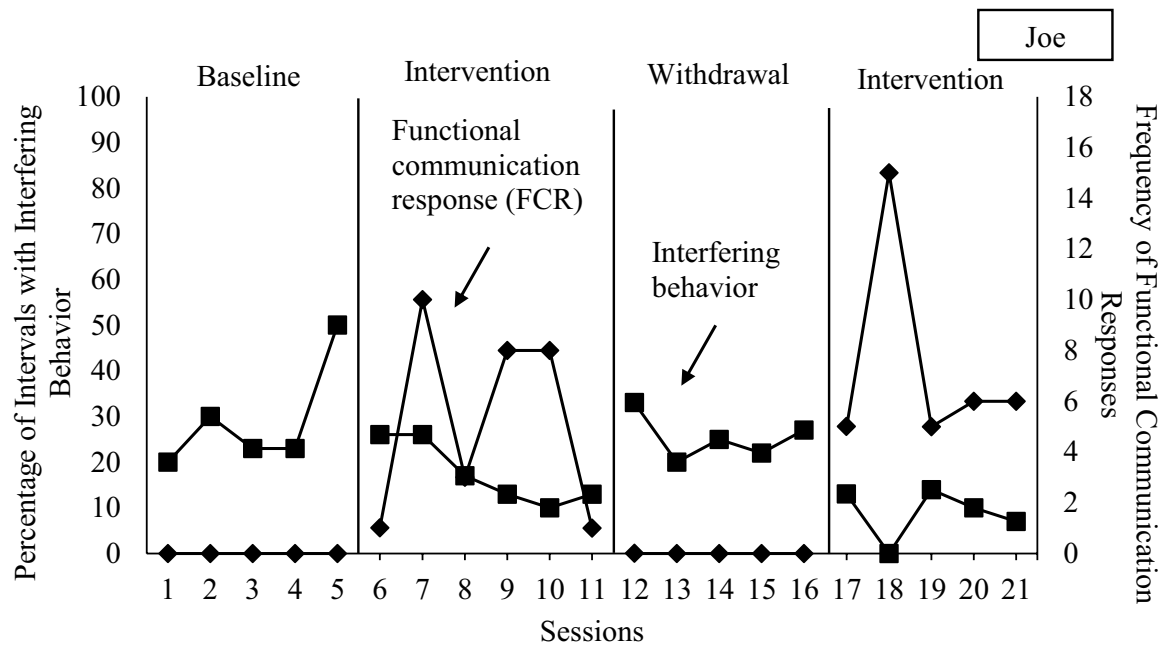

Fig. 1 This graph depicts the percentage of intervals with interfering behavior and frequency of FCRs for Joe

\section{Results}

\section{Joe and Gina}

During baseline, Gina's use of behavior strategies was $9 \%$ for each session. After BPT, Gina used an average of $92.1 \%$ intervention components (range $=87-100 \%$ ). Figure 1 displays both Joe's interfering behavior (percentage of 10-s partial intervals) and frequency of FCRs (mands for help, more food, and attention). During baseline, Joe exhibited a somewhat variable level of interfering behavior $(M=29.2 \%$; range $=20-50 \%$ ). During intervention, Joe's interfering behavior showed a decreasing trend with two overlapping data points $(M=17.5 \%$; range $=10-26 \%)$. When the intervention was removed, Joe's interfering behavior returned to baseline levels $(M=25.4 \%$; range $=20-33 \%)$ with increased stability, a change in trend, and two overlapping data points with the previous intervention condition. The intervention was reintroduced, and Joe's interfering behavior showed an immediate decrease in level with no overlapping data points $(M=8.8 \%$; range $=0-14 \%)$. There were three demonstrations of the basic effect and a functional relation was detected.

Joe's appropriate replacement behavior was the use of FCRs to mand for attention, mand for more food items, and mand for assistance with eating. During baseline, Joe did not engage in any FCRs. During intervention, there was an immediate (within two data points) change in level and trend. Joe engaged in highly variable instances of FCR $(M=5.2$; range $=1-10)$. During second baseline, Joe did not engage in any FCRs. Once the intervention was reintroduced, Joe engaged in slightly less variable responding with one outlying data point that corresponded with a novel stimulus of the rotating tray on the table $(M=7.4$; range $=5-15)$. There was a 
functional relation observed for FCRs. Joe and Gina participated in 21 baseline and intervention sessions for a duration of 59 days.

\section{Lucas and Catie}

During baseline Catie correctly implemented $12.8 \%$ (range $=7-22 \%$ ) of BSP steps. After BPT, she correctly completed $87.4 \%$ (range $=80-91 \%$ ) of steps. Figure 2 depicts Lucas interfering behavior (10 s partial intervals), frequency of FCRs (count), and task analysis steps completed for room cleaning (percent). Lucas' interfering behavior was high and variable during baseline $(M=71.6 \%$; range $=50$ $-93 \%)$. Once the intervention was implemented, there was an immediate decrease in level and a decreasing trend during the intervention phase $(M=20.6 \%$; range $=5$ $-33 \%$ ). When the intervention was withdrawn there was a change in trend and level with one data point overlapping between intervention and second baseline $(M=34.6 \%$; range $=32-40 \%)$. Once the intervention was implemented again, Lucas interfering behavior showed a change in level with one overlapping data point between intervention and second baseline $(M=22.6 \%$; range $=15-40 \%)$.

Lucas appropriate replacement behavior was the use of FCRs to mand for assistance with cleaning tasks or a break. During baseline, he used no FCRs. Upon implementation of the intervention, he used three FCRs for each session for assistance (maximum of three allowed). Once the intervention was withdrawn, he used zero FCRs. Upon the reintroduction of the intervention, Lucas used all three of his mands for help during each session.

For task analysis steps completed, Lucas engaged in correct responding that was variable during baseline with a slight increasing trend $(M=34.2 \% ; 18-50 \%)$. During intervention, Lucas engaged in higher percentage of room cleaning tasks (putting away laundry, sweeping the floor, making his bed) with an immediate increase in level and change in trend. His task analysis levels also increased in stability during intervention $(M=96.2 \%$; range $=86-100 \%)$. When the intervention was withdrawn, task analysis percentage decreased immediately with a decreasing trend, increased variability, and no overlapping data points $(M=52.2 \%$; range $=16-72 \%)$. When the intervention plan was reimplemented, Lucas task analysis completion increased and became more stable with an immediate change in level and trend to $100 \%$ completion across all five data points. Lucas and Catie participated in 20 baseline and intervention sessions for a duration of 57 days.

\section{Henry and Daniel}

During baseline, Daniel implemented $11 \%$ of intervention steps. After BPT, Daniel's implementation increased to an average of $88.2 \%$ (range $=84-93 \%$ ). Figure 3 depicts Henry's challenging behavior (10 s partial intervals), frequency of FCRs (mand for breaks), and number of seconds with toothbrush in mouth (total duration). Henry's challenging behavior was variable and increasing in baseline $(M=71 \%$; range $=53-87 \%)$. During intervention, Henry's challenging behavior decreased with an immediate change in level and trend with zero overlap. Henry's 

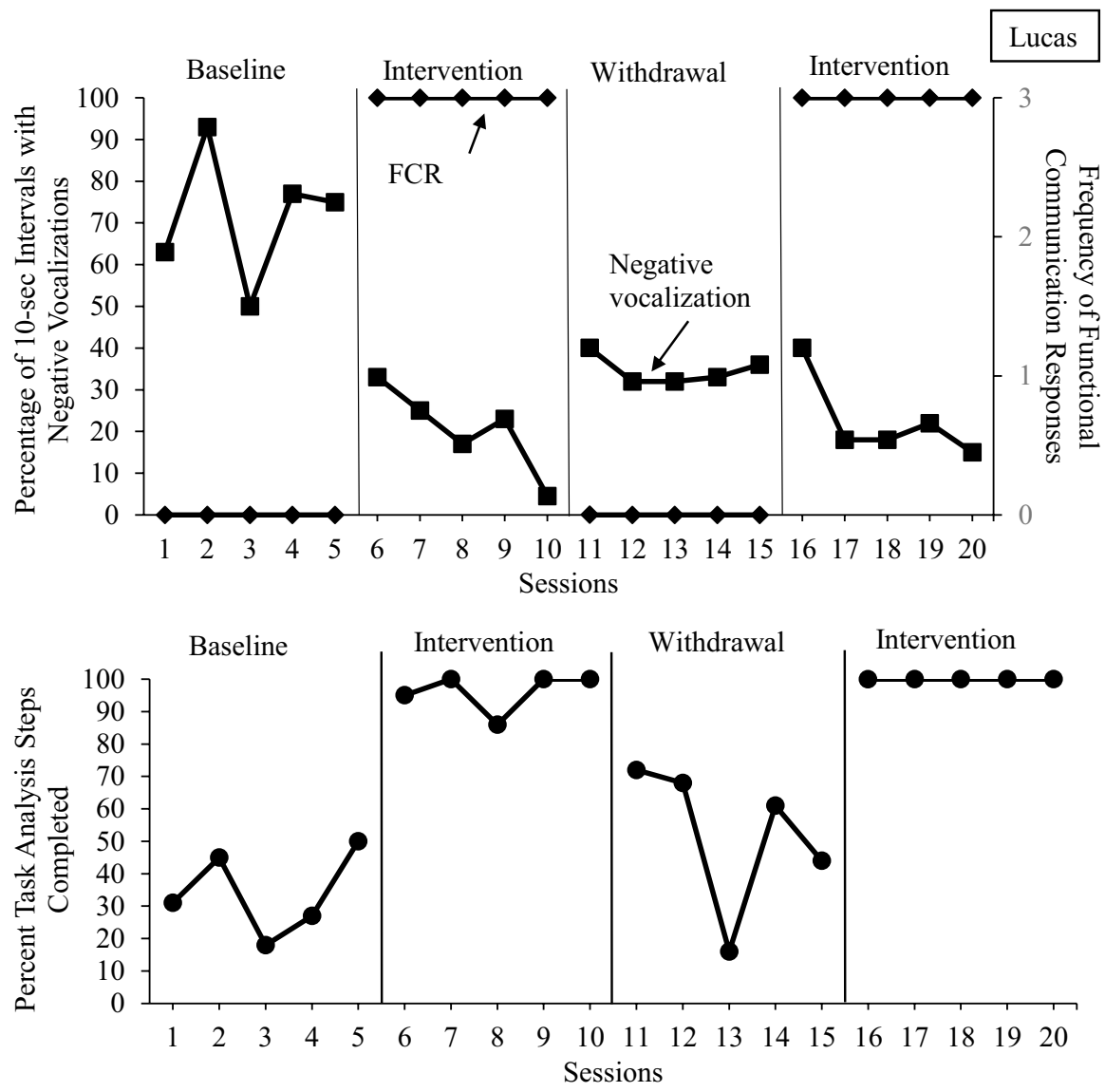

Fig. 2 The top panel depicts the percentage of intervals with interfering behavior and frequency of FCRs for Lucas. The bottom panel represents the percent of task analysis steps completed independently by Lucas

challenging behavior in the first intervention phase showed a decreasing trend and less variability than baseline $(M=24.2 \%$; range $=13-40 \%)$. When the intervention was removed, Henry's challenging behavior immediately increased with zero overlap and a change in trend and level $(M=84.6 \% ; 70-90 \%)$. With the reintroduction of the intervention, Henry's challenging behavior decreased in level with no overlapping data points and similar levels to the previous intervention phase $(M=35.6 \%$; range $=17-46 \%$ )

Henry's appropriate replacement behavior was the use of FCRs for a break for the task of toothbrushing. During baseline, Henry engaged in no FCRs. During the first intervention phase, Henry engaged in increased FCRs $(M=2.8$; range $=2-3)$. When the intervention was withdrawn, Henry returned to baseline levels of FCRs. With the reintroduction of the intervention, Henry engaged in a more variable level of FCRs $(M=1.8$; range $=1-4)$. Figure 3 also depicts the number of seconds Henry 

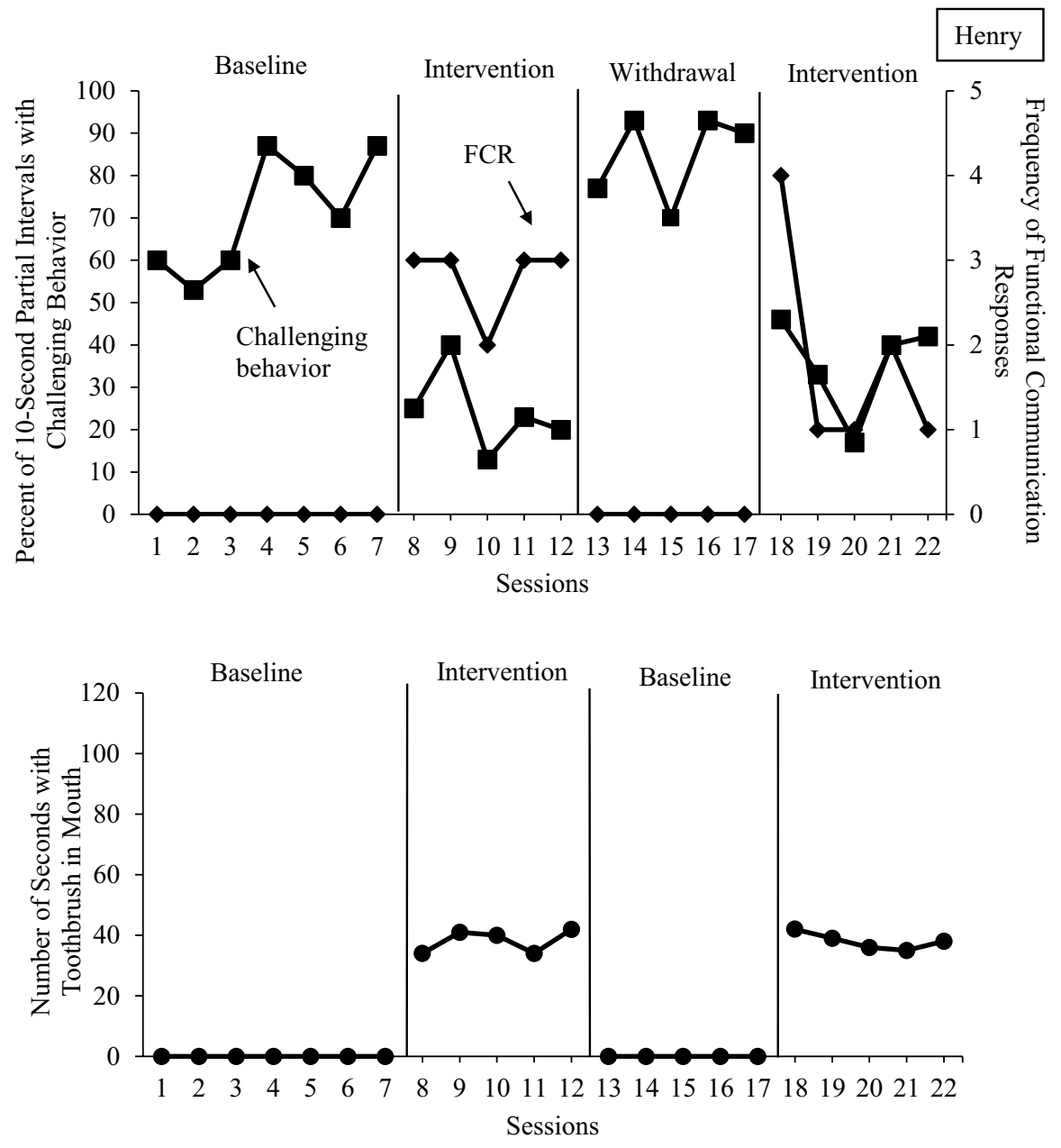

Fig. 3 The top panel represents percentage of intervals of challenging behavior and frequency of FCRs for Henry. The bottom panel represents the seconds that Henry kept the tooth brush in his mouth

opened his mouth so the toothbrush could be moved into and around the inside of his mouth (total duration). A terminal goal for the duration of toothbrushing was set at $120 \mathrm{~s}$. This is the dentist-recommended duration for toothbrushing for children and adults. During baseline, Henry did not open his mouth at all and the toothbrush was placed in his mouth for $0 \mathrm{~s}$. During intervention, the number of seconds the toothbrush remained in Henry's mouth increased immediately and remained stable with no overlap $(M=38.2$; range $=34-42)$. During second baseline, Henry did not open his mouth at all ( $0 \mathrm{~s}$ duration). During the second intervention phase, Henry increased total time the toothbrush remained in his mouth immediately with a change in level and trend $(M=38$; range $=35-42)$. Henry and Daniel participated in 22 baseline and intervention sessions for a duration of 55 days. 


\section{TARF-R Scores}

For each dyad, TARF-R assessments were conducted pre- and post- intervention. Parents rated each intervention as having good contextual fit $(\mathrm{M}=4.6$, range $=4.33-5)$, being highly acceptable $(M=4.9$, range $=4.5-5)$ and effective $(M=4.6$, range $=4.2-5)$, and having few disadvantages $(M=1.67$, range $=1-2.33)$.

\section{Discussion}

This study evaluated the effect of BPT and telehealth coaching on parent implementation of function-based BSPs, the effects of parent-implemented BSPs on the challenging and adaptive behavior of older children and adolescents with ID using an A-B-A-B design and assessed the acceptability of the BSPs pre- and post-intervention. The findings of this study extend the current literature in several meaningful ways.

First, adolescents and older children were selected for participation to address the gap in the literature on parent-implemented behavior interventions via telehealth for this age range (Gerow et al., 2018). The specific challenges presented by this population include extended learning history, potential worsening of challenging behavior in adolescence, physical growth spurts, and hormonal changes which may be associated with challenging behavior (Rattaz et al., 2018). During functional assessment interviews, each of the parents included in this study noted that the challenging behavior targeted in this study were long-standing including some from early childhood, and that they had not been adequately addressed by previous interventions including behavior plans, parent training, and school-based communication training.

Second, this study contributes to a growing body of research supporting the use of telecommunication technology to deliver effective intervention. Using consumerready videoconferencing equipment, the researcher was able to effectively conduct interviews, train and coach parents via bug-in-ear, and observe the routines of concern. Gina noted that the family could not have received effective behavioral support without telehealth based on their physical distance from behavior analysts. Daniel and Catie noted that even though telehealth required some troubleshooting of camera placement, they felt that the telehealth delivery method was more convenient, lessened their stress around cleaning their home prior to sessions, and allowed for more flexible scheduling.

Third, social validity was assessed and parent treatment fidelity was tracked for all participants, which is an area of need for parent-implemented behavior interventions (Gerow et al., 2018). Parents said they felt "very supported" during implementation and that the procedures were easy to understand.

As with all telehealth research, there are ethical implications for intervening on potentially harmful challenging behavior when the professional cannot implement any crisis protocols or safety plans. This is something not often mentioned in research which means practitioners are left with little guidance on how and when 
to implement safety plans and termination criteria. As it stands, there is very little information for ABA practitioners on the use of telehealth and this has potentially led to loss of services for individuals with IDD since Covid-19 has made in person services more difficult or impossible to provide. The use of termination criteria for one participant (Henry) provides one example of how these criteria can be used in telehealth service provision. Precautions such as creating safety plans for all behavior interventions, but particularly for those that include extinction or planning interventions that do not rely upon extinction may help addresses these barriers.

For each dyad, there are considerations for variables that potentially influenced the effectiveness for the intervention plan. For Joe in particular, several variables may have contributed to the intervention being slightly less effective in comparison to other dyads. First, FCRs did not correspond with changes in interfering behavior during the first intervention phase. This could be due to changes in motivating operations such as the relative availability of Gina's attention or Joe's need for more food items which fluctuated based on how much food he had on his plate at the beginning of the meal. The researcher hypothesized that Joe may have wanted assistance with eating, but he never manded for help independently. This could be because eating with his hands had a lower response effort than pressing a button and waiting for Gina to help with the use of the fork. Joe may have benefitted from the addition of a token economy or another reinforcement-based consequence intervention to ensure that the frequency of eating with his hands remains low by having the use of his fork, while effortful, match the reinforcement available. There was one outlier data point for FCRs for Joe that corresponded with the presence of many novel foods and a rotating tray on the table. Gina had made Joe pasta (at his request), while the rest of the family had a Greek meal in which many novel foods were presented. Joe manded using the iPad and then pointed to items on the rotating tray but did not touch the items. This is an excellent example of a naturally occurring motivating operation change. Introducing novel foods to Joe could increase his manding through environmental arrangement (in sight but out of reach), which is a very common antecedent manipulation and relatively easy for parents to implement (Wong et al., 2015).

\section{Limitations and Future Research}

Lucas interfering behavior did not completely resolve, even with effective use of FCRs. Analysis may have benefitted from a tiered intensity data collection method or the use of decibels as a measure of yelling. These methods may have given additional dimension to the data because during baseline, Lucas would often scream loudly and threaten, but during intervention most of his interfering behavior was grumbling and making sarcastic/negative comments. While these behaviors are not ideal, they are more age-appropriate than screaming and threatening and may be within social norms. Without the inclusion of typically developing age normed data on this routine, it is difficult to assess the appropriate level of less severe interfering behavior. Future research should include typically developing normative peer 
samples to determine what is an acceptable level of grumbling about unpreferred tasks for a teenager.

There are a few limitations of this project related to BSP development. First, the functions addressed in the BSP are a result of an interview (FAI - adolescent) and an indirect assessment tool (QABF), which may have led to incorrect or incomplete function identification. Second, the author did not use systematic decision rules governing how much the BSPs were modified based on parent input. The researcher accepted small changes from parent feedback and used clinical judgement to ensure that the BSP still addressed the function of the challenging behavior hypothesized based on the results of the QABF and FAI (Matson \& Vollmer, 1995; O’Neil et al., 1997). For example, Henry's parents prioritized moving the toothbrushing routine from his bedroom into the bathroom rather than prioritizing achieving the dentist-recommended two minutes of brushing during the intervention. Creating decision rules will increase the replicability, reproducibility, and potential manualization of BSP development for future research and practice. Future research should be transparent about these processes and strive to systematize the decisions.

This project also did not track the frequency or type of prompts provided by the researcher to each parent. Future research should track the specific types and frequency of prompts required to maintain treatment fidelity. Researchers should also track and types and frequency of errors made by parents during each session and the effect of withdrawing or systematically fading bug-in-ear coaching and the researcher's presence. This can be achieved by having the parents record sessions without the researcher present to assess independent performance. The removal of FCR stimuli and reinforcement available for appropriate communication may have resulted in artificially decreased FCRs and this is a limitation to the findings. Future research could leave the stimuli in place to determine if FCR use decreases immediately or follows a pattern of extinction responding and then decreases.

Other family members were not included in the intervention (siblings and other parents). They were provided information about the purpose of the study by the parent who served as the interventionist, but they were not required to behave in a particular way. Two families included young children, and they would occasionally enter the room where the intervention was taking place (bathroom; bedroom) and the dinner routine included all four family members. These variables were not controlled for and may increase the likelihood of successful generalization. Future research should address family member involvement if appropriate for the routine including training siblings as communication partners and assessing social validity for siblings and other family members as part of research. There are ethical concerns with involving siblings in research on challenging and interfering behavior such as exposure to verbal or physical aggression (Lucas and Henry), which should be addressed in future research.

One issue with BPT is high attrition. Facilitators of BPT that increase participation are: supportive and individualized feedback from professionals, increased accessibility, affordability, flexibility, and social-emotional support and connection with a community (Raulston et al., 2019). The current study provided individualized and supportive feedback, used telehealth to address transportation and distance barriers, 
was free to the participants, and had flexible scheduling to meet the needs of other family obligations. Telehealth also enabled service provision in family homes which can ameliorate barriers parents face living in rural areas or areas where specialist availability is limited.

Generalization was not assessed for these interventions. Future research should also include generalization outside of the intervention environment to socially significant routines and locations where appropriate (e.g., going out to eat for Joe and Gina and brushing teeth at Grandma's house for Henry).

\section{Declarations}

Conflict of interest We have no known conflict of interest to disclose.

Informed consent Each parent signed informed consent prior to participation in this study.

\section{References}

Alnemary, F. M., Wallace, M., Symon, J. B., \& Barry, L. M. (2015). Using international videoconferencing to provide staff training on functional behavioral assessment. Behavioral Interventions, 30(1), 73-86.

Dib, N., \& Sturmey, P. (2012). Behavioral skills training and skill learning. In N. M. Seel (Ed.), Encyclopedia of the sciences of learning. USA: Springer.

Emerson, E., Kiernan, C., Alborz, A., Reeves, D., Mason, H., Swarbrick, R., Mason, L., \& Hatton, C. (2001). The prevalence of challenging behaviors: A total population study. Research in Developmental Disabilities, 22(1), 77-93.

Gentry, J. A., \& Luiselli, J. K. (2008). Treating a child's selective eating through parent implemented feeding intervention in the home setting. Journal of Developmental and Physical Disabilities, 20(1), 63-70.

Gerow, S., Hagan-Burke, S., Rispoli, M., Gregori, E., Mason, R., \& Ninci, J. (2018). A systematic review of parent-implemented functional communication training for children with ASD. Behavior Modification, 42(3), 335-363. https://doi.org/10.1177/0145445517740872

Hanley, G. P., Jin, C. S., Vanselow, N. R., \& Hanratty, L. A. (2014). Producing meaningful improvements in problem behavior of children with autism via synthesized analyses and treatments. Journal of Applied Behavior Analysis, 47(1), 16-36. https://doi.org/10.1002/jaba.106

Hilty, D. M., Ferrer, D. C., Parish, M. B., Johnston, B., Callahan, E. J., \& Yellowlees, P. M. (2013). The effectiveness of telemental health: A 2013 review. Telemedicine and e-Health, 19(6), 444-454. https://doi.org/10.1089/tmj.2013.0075

Kogan, M. D., Strickland, B. B., Blumberg, S. J., Singh, G. K., Perrin, J. M., \& van Dyck, P. C. (2008). A national profile of the health care experiences and family impact of autism spectrum disorder among children in the United States, 2005-2006. Pediatrics, 122, 1149-1158. https://doi.org/10.1542/peds. 2008-1057

Ledford, J. R., \& Gast, D. L. (2018). Single case research methodology: Applications in special education and behavioral sciences. Routledge. https://doi.org/10.4324/9781315150666

Lee, L. C., Harrington, R. A., Louie, B. B., \& Newschaffer, C. J. (2008). Children with autism: Quality of life and parental concerns. Journal of Autism and Developmental Disorders, 38(6), 1147-1160. https://doi.org/10.1007/s10803-007-0491-0

Lindgren, S., Wacker, D., Suess, A., Schieltz, K., Pelzel, K., Kopelman, T., Lee, J., Romani, P., \& Waldron, D. (2016). Telehealth and autism: Treating challenging behavior at lower cost. Pediatrics, 137(Supplement 2), S167-S175. https://doi.org/10.1542/peds.2015-2851o 
Lowe, K., Allen, D., Jones, E., Brophy, S., Moore, K., \& James, W. (2007). Challenging behaviours: Prevalence and topographies. Journal of Intellectual Disability Research, 51(8), 625-636. https:// doi.org/10.1111/j.1365-2788.2006.00948.x

Machalicek, W., Lequia, J., Pinkelman, S., Knowles, C., Raulston, T., Davis, T., \& Alresheed, F. (2016). Behavioral telehealth consultation with families of children with autism spectrum disorder. Behavioral Interventions, 31(3), 223-250. https://doi.org/10.1002/bin.1450

Machalicek, W., Raulston, T., Drew, C., \& Ruppert, T. (2015). Telehealth behavioral consultation with families of children with autism. The international society for the study of behavioural development (ISSBD) Bulletin Special Section on Autism Spectrum Disorder, 39(6), 9-14. https://doi.org/ 10.1002/bin. 1450

Matson, J. L. (2012). Functional assessment for challenging behaviors. Springer. https://doi.org/10.1007/ 978-1-4614-3037-7

Matson, J. L., \& Neal, D. (2009). Psychotropic medication use for challenging behaviors in persons with intellectual disabilities: An overview. Research in Developmental Disabilities, 30(3), 572-586.

Matson, J. L., \& Vollmer, T. R. (1995). User's guide: Questions about behavioral function (QABF). Scientific Publishers. https://doi.org/10.1037/t64275-000

Mello, M. P., Goldman, S. E., Urbano, R. C., \& Hodapp, R. M. (2016). Services for children with autism spectrum disorder: Comparing rural and non-rural communities. Education and Training in Autism and Developmental Disabilities, 1, 355-365.

O’Dwyer, C., McCallion, P., Burke, É., Carroll, R., O’Dwyer, M., \& McCarron, M. (2018). Prevalence and associated factors of problem behaviours among older adults with intellectual disabilities in Ireland. Research in developmental disabilities, 80, 192-204. https://doi.org/10.1016/j.ridd.2018.05.007

Rattaz, C., Michelon, C., Munir, K., \& Baghdadli, A. (2018). Challenging behaviours at early adulthood in autism spectrum disorders: Topography, risk factors and evolution. Journal of Intellectual Disability Research, 62(7), 637-649. https://doi.org/10.1111/jir.12503

Raulston, T. J., Hieneman, M., Caraway, N., Pennefather, J., \& Bhana, N. (2019). Enablers of behavioral parent training for families of children with autism spectrum disorder. Journal of Child and Family Studies, 28(3), 693-703. https://doi.org/10.1007/s10826-018-1295-X

Reimers, T. M., \& Wacker, D. P. (1988). Parents' ratings of the acceptability of behavioral treatment recommendations made in an outpatient clinic: A preliminary analysis of the influence of treatment effectiveness. Behavioral Disorders, 14(1), 7-15. https://doi.org/10.1177/019874298801400104

Simó-Pinatella, D., Mumbardó-Adam, C., Alomar-Kurz, E., Sugai, G., \& Simonsen, B. (2019). Prevalence of challenging behaviors exhibited by children with disabilities: Mapping the literature. Journal of Behavioral Education, 28(3), 323-343.

Suess, A. N., Romani, P. W., Wacker, D. P., Dyson, S. M., Kuhle, J. L., Lee, S. D., Lindgren, T. G., Kopelman, K. E., \& Pelzel Waldron, D. B. (2014). Evaluating the treatment fidelity of parents who conduct in-home functional communication training with coaching via telehealth. Journal of Behavioral Education, 23(1), 34-59. https://doi.org/10.1007/s10864-013-9183-3

Unholz-Bowden, E., McComas, J. J., McMaster, K. L., Girtler, S. N., Kolb, R. L., \& Shipchandler, A. (2020). Caregiver training via telehealth on behavioral procedures: A systematic review. Journal of Behavioral Education, 29(2), 246-281.

Wacker, D. P., Lee, J. F., Dalmau, Y. C. P., Kopelman, T. G., Lindgren, S. D., Kuhle, J., Pelzel, K. E., Dyson, S., Schieltz, K. M., \& Waldron, D. B. (2013). Conducting functional communication training via telehealth to reduce the problem behavior of young children with autism. Journal of Developmental and Physical Disabilities, 25(1), 35-48. https://doi.org/10.1007/s10882-012-9314-0

Wong, C., Odom, S. L., Hume, K. A., Cox, A. W., Fettig, A., Kucharczyk, S., Brock, M. E., Plavnick, J. B., Fleury, V. P., \& Schultz, T. R. (2015). Evidence-based practices for children, youth, and young adults with autism spectrum disorder: A comprehensive review. Journal of Autism and Developmental Disorders, 45(7), 1951-1966. https://doi.org/10.1007/s10803-014-2351-z

Publisher's Note Springer Nature remains neutral with regard to jurisdictional claims in published maps and institutional affiliations. 